\title{
Diseño y Validación de un Cuestionario para el Diagnóstico de Riesgos Psicosociales en Empresas Ecuatorianas
}

\author{
DESIGN AND VALIDATION OF A QUESTIONNAIRE FOR THE DIAGNOSIS OF PSYCHOSOCIAL RISKS IN \\ ECUATORIAN COMPANIES
}

Lars T. Moreno Alestedt ${ }^{1}$, Santiago M. Vaca Morales², Diana I. Martínez Changuan³ ${ }^{3}$ Pablo R. Suasnavas Bermúdez ${ }^{4}$ Isabel M. Cárdenas

Moncayo ${ }^{5}$, Antonio R. Gómez Garcia ${ }^{6}$

1. Ministerio de Salud Pública, Quito, Ecuador. larsmoreno@yahoo.com

2. Instituto Ecuatoriano de Seguridad Social, Quito, Ecuador. santiago.vaca@hotmail.es

3. Ministerio de Salud Pública, Quito, Ecuador. i.diana.martinez@gmail.com

4. Universidad Internacional SEK, Quito, Ecuador. pablo.suasnavas@uisek.edu.ec

5. Universidad de Especialidades Espíritu Santo, Guayaquil, Ecuador. isacardenasm@yahoo.com

6. Universidad Internacional SEK, Quito, Ecuador. antonio.gomez@uisek.edu.ec

\begin{abstract}
RESUMEN
Objetivo: presentar el diseño, construcción y validación de un cuestionario para el diagnóstico de riesgos psicosociales en Ecuador, conformado por 58 items que evalúan el riesgo psicosocial a través de 8 dimensiones. Diseño: estudio transversal en 2 fases: (i) diseño del marco conceptual y construcción del cuestionario y; (ii) valoración de la validez y consistencia interna aplicado en una muestra de 3.225 trabajadores de empresas ecuatorianas. Resultados: El cuestionario posee una alta fiabilidad en términos de consistencia interna (Alfa de Cronbach $=0,968$ ) y una adecuación satisfactoria de los datos a la matriz factorial $(\mathrm{KMO}=0,980$; Bartlett $=93818,0,<0,05)$. Conclusiones: se recomienda el uso de este cuestionario como instrumento para el diagnóstico preliminar, permitiendo el diseño e implementación de futuros programas de prevención de riesgos psicosociales en empresas de más de 10 trabajadores en el país.
\end{abstract}

(Moreno L, Vaca S, Martínez D, Suasnavas P, Cárdenas I, Gómez A, 2018. Diseño y Validación de un Cuestionario para el Diagnóstico de Riesgos Psicosociales en Empresas Ecuatorianas. Cienc Trab. SepDic; 20 [63]: 160-168).

Palabras clave: CUESTIONARIO; DIAGNOSTICO; RIESGO PSICOSOCIAL; TRABAJADORES; ECUADOR.

\section{ABSTRACT}

Objective: to present the design, construction and validation of a questionnaire for the diagnosis of psychosocial risks in Ecuador, consisting of 58 items that assess psychosocial risk through 8 dimensions. Design: cross-sectional study in 2 phases: (i) design of the conceptual framework and construction of the questionnaire and; (ii) assessment of the validity and internal consistency applied to a sample of 3.225 workers from ecuadorian companies. Results: The questionnaire has a high reliability in terms of internal consistency (Cronbach's alpha $=0,968$ ) and a satisfactory adaptation of the data to the factorial matrix $(\mathrm{KMO}=0,980$; Bartlett $=93818,0,<0,05)$. Conclusions: the use of this questionnaire is recommended as an instrument for preliminary diagnosis, allowing the design and implementation of future psychosocial risk prevention programs in companies with more than 10 workers in the country.

Key Words: QUESTIONNAIRE; DIAGNOSIS; PSYCHOSOCIAL RISK; WORKERS; ECUADOR.

\section{INTRODUCCIÓN}

Los factores psicosociales consisten en interacciones entre, por una parte, el trabajo, el medio ambiente y las condiciones de organización, y por la otra, las capacidades del trabajador, sus

\section{Correspondencia / Correspondence:}

Ph.D. Antonio Ramón Gómez García

Universidad Internacional SEK. Campus Miguel de Cervantes

Alberto Einstein, s/n y 5 ta transversal

Quito, Ecuador.

Tel.: +593 3974800 Ext. 169

e-mail: antonio.gomez@uisek.edu.ec

Recibido: 22 de octubre de 2018 / Aceptado: 28 de noviembre de 2018 necesidades, su cultura y su situación personal fuera del trabajo, todo lo cual, a través de percepciones y experiencias, pueden influir en la salud, el rendimiento y la satisfacción en el trabajo. ${ }^{1,2}$ Por tanto, una cuestión central en prevención y promoción de la salud está constituida por la identificación de aquellos factores de riesgo psicosocial que permitan intervenciones precisas para mejorar las condiciones de trabajo y estado de salud en la población trabajadora.

Son numerosos los instrumentos de evaluación de riesgos psicosociales desarrollados y aplicados a nivel mundial ${ }^{3-6}$ y en Latinoamérica. ${ }^{2,7-11} \mathrm{Si}$ bien en Ecuador se han realizado varios estudios para medir estos factores de riesgo ${ }^{12,13}$, aún sigue siendo escaso el conocimiento de esta problemática y además todos ellos han empleado instrumentos validados en otros países. Como menciona Pando y colaboradores ${ }^{2}$, existe la necesidad de contar con instrumentos de medición de riesgos psicosociales ajustados 
al contexto y que recojan información desde los trabajadores ${ }^{15} \mathrm{y}$, que a su vez sean confiables y válidos. ${ }^{16}$

Por tanto, en relación a los antecedentes anteriormente expuestos y su pertinencia, esta investigación tiene por objetivo presentar el proceso de diseño, construcción y validación de un cuestionario fiable y de fácil manejo para el diagnóstico de riesgos psicosociales en empresas ecuatorianas de más diez trabajadores que permita implementar futuros programas de prevención. Igualmente se muestran los resultados de la población trabajadora encuestada según características socio-demográficas, laborales, de empleo y nivel de riesgo global.

\section{MATERIAL - MÉTODO}

Se trata de un estudio descriptivo, observacional de corte transversal desarrollado en 2 fases: (i) diseño del marco conceptual y construcción del cuestionario y; (ii) valoración de la validez y consistencia interna del mismo para el diagnóstico de riesgos psicosociales en empresas e instituciones públicas y privadas, cuyo organismo ejecutor fue la el Ministerio del Trabajo a través de la Dirección de Seguridad, Salud en el Trabajo y Gestión Integral de Riesgos entre los meses de marzo a noviembre del 2017.

\section{Fase I. Diseńo del marco conceptual y construcción del cuestionario}

Para diseñar el marco conceptual (dimensiones e ítems) se revisaron teorías, estudios e instrumentos similares disponibles en la literatura científica ${ }^{2-16}$, a través de la búsqueda y recuperación en bases de datos internacionales, así como la normativa legal vigente del país. ${ }^{17-21}$ En una primera versión del cuestionario se establecieron 83 items agrupados en 8 dimensiones.

Entre los meses de junio y julio, el Ministerio del Trabajo convoco en dos mesas de trabajo consecutivas a expertos nacionales en la temática (empresas e instituciones públicas - privadas y universidades) para la adecuación y validación de las dimensiones e items de la primera versión, así como, el grado de comprensión y adecuación al contexto sociocultural del país. Se obtuvo la versión definitiva del cuestionario (58 ítmes): Carga y Ritmo de Trabajo (4 items); Desarrollo de Competencias (4 items); Liderazgo (6 items); Margen de Acción y Control (4 items); Organización del Trabajo (6 items); Recuperación (5 items) y; Soporte y Apoyo (5 items), además otros aspectos: Acoso Discriminatorio (4); Acoso Laboral (2); Acoso Sexual (2); Adicción al Trabajo (5); Condiciones de Trabajo (2); Doble Presencia (2); Estabilidad Laboral y Emocional (5) y; Salud Auto-percibida (2), ver Anexo 1.

Cada item del cuestionario consta de 4 opciones de respuesta $(4=$ Completamente de acuerdo, 3 = Parcialmente de acuerdo, 2 = Poco de acuerdo y 1 = En desacuerdo), a menor puntuación mayor riesgo psicosocial. ${ }^{16}$ La suma de los ítems por dimensión así como la suma de todos los ítems del cuestionario agrupados por terciles permite obtener tres rasgos de riesgo psicosocial, el resultado global es la suma de todas las dimensiones: Riesgo Bajo (puntuaciones de 175 a 232) efecto mínimo sobre la seguridad y salud, no genera a corto plazo efectos nocivos; Riesgo Medio (puntuaciones de 117 a 174) efecto moderado y que puede generar a mediano plazo efectos nocivos para la salud, afectaciones a la integridad física y posibles enfermedades profesionales y; Riesgo Alto (puntuaciones de 58 a 116) impacto potencial efecto alto e inmediato, ver Anexo 2 y 3.

\section{Fase II. Validez y consistencia interna del cuestionario}

El universo está compuesto por las empresas e instituciones públicas y privadas con más de 10 empleados, pertenecientes a todas las ramas de actividad económicas y legamente constituidas en el país. Estas se encuentran desagregadas por todas las actividades económicas, de acuerdo a las bases de datos que cuenta el Ministerio del Trabajo para sus procesos de inspección, como es el Directorio de Empresas de $2016^{22}$ del Instituto Nacional de Estadística y Censos, Portal de información / sector societario de la Superintendencia de Compañías, fuentes disponibles de información pública y actualizada a la fecha de realización del diseño muestral. La selección de las empresas dentro de cada provincia del país se realizó de forma sistemática y aleatoria, mediante el muestreo aleatorio simple de forma que la proporcionalidad entre las actividades productivas ${ }^{23}$ y tamaño de las empresas por número de personas trabajadoras ${ }^{24} y$ fuesen representativas $(\mathrm{n}=385)$.

A la empresa y/o institución seleccionada se le comunicó vía telefónica la participación voluntaria al estudio garantizando la confidencialidad, aceptada la participación se remitió mediante correo electrónico el oficio circular de invitación formal. Es importante mencionar que en algunos casos no hubo respuesta y/o negación, por lo que se optó por comunicarse con la segunda empresa y/o institución según el marco muestral.

Para cada una de las empresas y/o instituciones seleccionadas y en función a su número total de personas trabajadoras se aplicó un muestreo probabilístico, no proporcional y representativo (nivel de confianza del 95\% y un margen de error del $\pm 5 \%$ ) permitiendo determinar el tamaño muestral final ( $\mathrm{n}=4.346$ encuestas).

Previo al trabajo de campo para la aplicación de la encuesta se procedió a capacitar a los analistas de seguridad y salud en el trabajo (11) y analistas de atención de grupos prioritarios (9) del Ministerio del Trabajo, quienes acudieron personalmente a las respectivas empresas e instituciones para la aplicación del cuestionario y recogida de información durante los meses de julio, agosto, septiembre del 2017.

Finalizada esta fase, los cuestionarios fueron tabulados en la aplicación Excel de Microsoft Office y sometidos a un proceso de validación y depuración para la detección y corrección de errores mediante el programa estadístico Statistical Package for the Social Sciences (SPSS, versión 25) para Windows, finalmente se validaron un total de 3.225 encuestas. Periódicamente se supervisó la aplicación de la encuesta por los responsables del Ministerio del Trabajo para garantizar la calidad de los datos. Los trabajadores encuestados fueron informados sobre los objetivos y finalidad del cuestionario y contestaron de manera auto-administrada in-situ en la propia empresa y/o institución. Para el caso de trabajadores con instrucción básica fue asistida por los analistas, se contó con el debido consentimiento de los participantes, el anonimato y el tratamiento adecuado de los datos personales.

Para evaluar la consistencia interna de las escalas de respuetas del cuestionario se empleó el valor Alfa de Cronbach para cada ítem por dimensión y total de ítems, considerando aceptables valores $\geq 0,600$ y buenos cuando eran $\geq 0,700 .{ }^{25,26}$

Previo al análisis factorial exploratorio ${ }^{27}$ (componentes principales con rotación Varimax) para evaluar la validez de constructo del cuestionario, se evaluó la adecuación del análisis factorial mediante las pruebas de Kaiser-Meyer-Olkin (KMO) y de esfericidad de Bartlett. Finalmente, se aplicó el Coeficiente de Correlación de Pearson $(\mathrm{p}<0.01)$ para conocer la asociación estadística para cada uno de los ítems del cuestionario. ${ }^{28}$ 


\section{RESULTADOS}

La población trabajadora encuestada para la validación del instrumento fue de 3.225 trabajadores (68\% fueron hombres y $32 \%$ mujeres) con una edad media de 35,4 (D.E. 9,6), siendo el grupo de edad más amplio entre los 25 a 43 años $(n=2.268$; 70\%) con estudios igual o superior al Bachillerato. El sector servicios e industria concentran la mayor parte de los trabajadores, principalmente de grandes empresas ( $\geq 100$ trabajadores) y con una antigüedad laboral superior a los 3 años ( $\mathrm{n}=2.300 ; 71 \%)$, Tabla 1 .

La distribución porcentual de los trabajadores encuestados de las empresas participantes según provincia: el 27,0\% en la provincia del Guayas ( $n=871$ ); el 23,7\% Pichincha ( $n=763)$; el 11,3\% Tungurahua $(n=363)$, el 8,2\% El Oro $(n=264)$; el 6,0\% Loja $(n=192)$; el $4,8 \%$ Manabí ( $n=154)$; el 4,4\% Imbabura ( $n=141)$; el 3,3\% Azuay ( $n=106)$; el 3,0\% Chimborazo ( $n=98)$; el 2,5\% Cotopaxi ( $n=82)$; el 2,4\% Napo $(\mathrm{n}=77)$; el 1,8\% Esmeraldas ( $\mathrm{n}=59)$; el 1,0\% Sucumbíos (n=32) y; el $0,7 \%$ en la provincia del Carchi $(n=23)$.

De los resultados del análisis se observa un nivel de riesgo bajo $(\mathrm{n}=2.378 ; 73,7 \%)$ en la mayoría de la población trabajadora encuestada, no obstante, un 24,4\% ( $n=786)$ y un $1,9 \%(n=61)$ se encuentran en un riesgo medio y alto respectivamente. Los niveles más altos los presentan aquellos trabajadores del sector agrícola e industrial, sin encontrar diferentes por grupos de edad y antigüedad laboral.

El resultado del análisis de fiabilidad global del instrumento mediante el empleo de Alfa de Cronbach fue de 0,968 para el total de ítems, apunta a una excelente consistencia interna, lo cual denota un nivel alto de precisión de las respuestas de los trabajadores encuestados y un alto grado de heterogeneidad en la muestra, Tabla 2.

No obstante, algunas dimensiones no superaron valores aceptables $(\alpha \geq 0,600)$, en particular, las sub-dimensiones de las Otras Dimensiones: Doble Presencia $(\alpha=0,490)$, Condiciones de Trabajo

Tabla 1.

Características socio-demográficas, laborales, de empleo y nivel de riesgo global de la población trabajadora $(n=3.225)$.

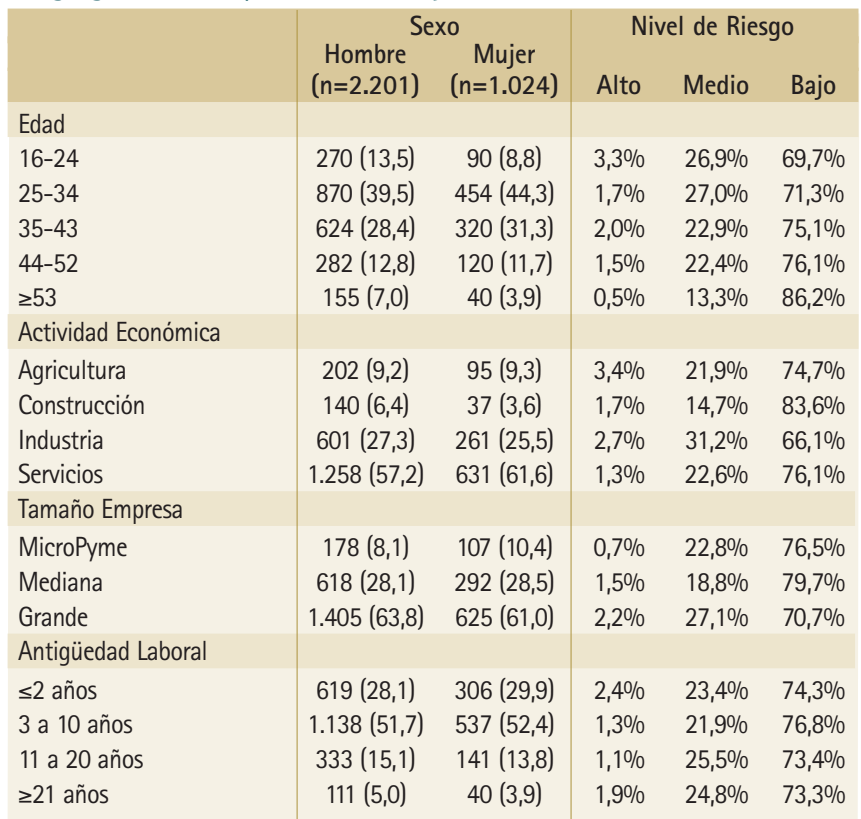

Tabla 2.

Análisis descriptivos, asimetría y curtosis de cada uno de los items y coeficientes alfa de Cronbach por dimensión.

\begin{tabular}{|c|c|c|c|c|c|c|}
\hline Dimensión & $\begin{array}{l}\text { İtem } \\
\mathrm{N}^{\circ}\end{array}$ & Media & $\begin{array}{l}\text { Desv. } \\
\text { Estándar }\end{array}$ & Asimetría & Curtosis & $\begin{array}{l}\text { Alfa de } \\
\text { Cronbach }\end{array}$ \\
\hline \multicolumn{7}{|l|}{ Carga y Ritmo de Trabajo } \\
\hline & 1 & 3,5 & 0,69 & $-1,29$ & 1,37 & \multirow[t]{4}{*}{0,714} \\
\hline & 2 & 3,3 & 0,84 & $-1,15$ & 0,66 & \\
\hline & 3 & 3,1 & 0,92 & $-0,76$ & $-0,31$ & \\
\hline & 4 & 3,2 & 0,89 & $-0,98$ & 0,13 & \\
\hline \multicolumn{7}{|c|}{ Desarrollo de Competencias } \\
\hline & 5 & 3,8 & 0,53 & $-2,62$ & 7,78 & \multirow[t]{4}{*}{0,667} \\
\hline & 6 & 3,6 & 0,72 & $-1,73$ & 2,55 & \\
\hline & 7 & 2,9 & 1,03 & $-0,53$ & $-0,90$ & \\
\hline & 8 & 3,2 & 0,96 & $-0,89$ & $-0,24$ & \\
\hline \multicolumn{7}{|l|}{ Liderazgo } \\
\hline & 9 & 2,9 & 1,05 & $-0,56$ & $-0,93$ & \multirow[t]{6}{*}{0,900} \\
\hline & 10 & 3,2 & 0,94 & $-0,98$ & $-0,07$ & \\
\hline & 11 & 3,3 & 0,89 & $-1,01$ & 0,15 & \\
\hline & 12 & 3,1 & 0,99 & $-0,82$ & $-0,47$ & \\
\hline & 13 & 3,2 & 0,93 & $-0,87$ & $-0,25$ & \\
\hline & 14 & 3,2 & 0,95 & $-0,91$ & $-0,24$ & \\
\hline \multicolumn{7}{|c|}{ Margen de Acción y Control } \\
\hline & 15 & 2,9 & 1,00 & $-0,51$ & $-0,84$ & \multirow[t]{4}{*}{0,823} \\
\hline & 16 & 3,4 & 0,82 & $-1,22$ & 0,77 & \\
\hline & 17 & 3,1 & 0,93 & $-0,85$ & $-0,20$ & \\
\hline & 18 & 3,3 & 0,92 & $-1,10$ & 0,20 & \\
\hline \multicolumn{7}{|l|}{ Organización del Trabajo } \\
\hline & 19 & 3,3 & 0,84 & $-1,03$ & 0,33 & \multirow[t]{6}{*}{0,836} \\
\hline & 20 & 3,1 & 0,97 & $-0,73$ & $-0,52$ & \\
\hline & 21 & 3,5 & 0,82 & $-1,55$ & 1,61 & \\
\hline & 22 & 3,1 & 0,93 & $-0,80$ & $-0,36$ & \\
\hline & 23 & 3,4 & 0,82 & $-1,17$ & 0,68 & \\
\hline & 24 & 3,7 & 0,67 & $-2,16$ & 4,55 & \\
\hline \multicolumn{7}{|l|}{ Recuperación } \\
\hline & 25 & 3,0 & 0,94 & $-0,64$ & $-0,51$ & \multirow[t]{5}{*}{0,846} \\
\hline & 26 & 3,1 & 0,99 & $-0,83$ & $-0,46$ & \\
\hline & 27 & 3,0 & 0,97 & $-0,60$ & $-0,67$ & \\
\hline & 28 & 3,3 & 0,91 & $-1,19$ & 0,46 & \\
\hline & 29 & 3,3 & 0,90 & $-1,07$ & 0,24 & \\
\hline Soporte y Apoyo & & & & & & \\
\hline & 30 & 3,3 & 0,83 & $-1,00$ & 0,33 & 0,796 \\
\hline & 31 & 3,2 & 0,89 & $-0,97$ & 0,05 & \\
\hline & 32 & 3,4 & 0,81 & $-1,39$ & 1,22 & \\
\hline & 33 & 3,3 & 0,87 & $-0,97$ & 0,09 & \\
\hline & 34 & 3,1 & 1,06 & $-0,86$ & $-0,60$ & \\
\hline Acoso Discriminatorio & & & & & & \\
\hline & 35 & 3,4 & 0,90 & $-1,27$ & 0,62 & 0,698 \\
\hline & 38 & 3,4 & 0,87 & $-1,34$ & 0,85 & \\
\hline & 53 & 3,6 & 0,69 & $-2,01$ & 3,68 & \\
\hline & 56 & 3,3 & 0,99 & $-1,18$ & 0,13 & \\
\hline Otras Dimensiones & & & & & & \\
\hline Acoso Laboral & 41 & 3,3 & 0,89 & $-1,10$ & 0,34 & 0,627 \\
\hline & 50 & 3,2 & 0,95 & $-0,95$ & $-0,13$ & \\
\hline Acoso Sexual & 43 & 3,6 & 0,77 & $-1,97$ & 3,16 & 0,687 \\
\hline & 48 & 3,7 & 0,73 & $-2,41$ & 5,14 & \\
\hline Adición al Trabajo & 36 & 3,5 & 0,72 & $-1,43$ & 1,73 & 0,703 \\
\hline & 45 & 3,4 & 0,81 & $-1,30$ & 1,07 & \\
\hline & 51 & 3,6 & 0,66 & $-1,86$ & 3,36 & \\
\hline & 55 & 3,2 & 0,90 & $-1,01$ & 0,14 & \\
\hline & 57 & 3,4 & 0,85 & $-1,21$ & 0,73 & \\
\hline Condiciones de Trabajo & 40 & 3,3 & 0,91 & $-1,08$ & 0,22 & 0,504 \\
\hline & 47 & 3,4 & 0,83 & $-1,43$ & 1,34 & \\
\hline Doble Presencia & 46 & 3,4 & 0,81 & $-1,45$ & 1,51 & 0,490 \\
\hline & 49 & 3,2 & 0,95 & $-1,06$ & 0,05 & \\
\hline Estabilidad Laboral & 37 & 3,3 & 0,88 & $-1,02$ & 0,19 & 0,832 \\
\hline & 39 & 3,4 & 0,82 & $-1,24$ & 0,75 & \\
\hline & 42 & 3,1 & 1,00 & $-0,89$ & $-0,37$ & \\
\hline & 52 & 3,7 & 0,66 & $-2,09$ & 4,13 & \\
\hline & 54 & 3,3 & 0,89 & $-1,15$ & 0,49 & \\
\hline Salud Auto-Percibida & 44 & 3,6 & 0,72 & $-1,73$ & 2,67 & 0,509 \\
\hline & 58 & 3,4 & 0,83 & $-1,24$ & 0,84 & \\
\hline
\end{tabular}


$(\alpha=0,504)$ y Salud Auto-Percibida $(\alpha=0,509)$, Tabla 2 . A pesar de estos valores, se observa que los ítems número 46 y 49 de la subdimensión Doble Presencia presentan una correlación significativa $(\mathrm{r}=0,329 ; \mathrm{p}<0,01)$, los items 40 y 47 de Condiciones de Trabajo $(\mathrm{r}=0,338 ; \mathrm{p}<0,01)$ y los ítems 44 y 58 de Salud AutoPercibida $(r=0,345 ; p<0,01)$. Asimismo, en la Tabla 3 se presentan las correlaciones por dimensiones del cuestionario.

Los resultados de las pruebas de KMO fue de 0,980 $(>0,50)$ y esfericidad de Bartlett de 93818,0 con una significancia de 0,000 $(<0,05)$, en ambos casos los resultados presentan valores favorables para continuar con el análisis factorial.

Los 58 ítems fueron distribuidos en 7 factores, presentando globalmente una varianza explicada de 53,0\%, aceptando valores superiores a 0,3 , Tabla 4 .

\section{DISCUSIÓN}

Diagnosticar los factores de riesgo psicosocial en las empresas ecuatorianas es una obligación legal y una necesidad prioritaria para realizar intervenciones que mejoren las condiciones de seguridad y salud en el trabajo. ${ }^{17-21,29}$

El Ministerio del Trabajo, en su afán de facilitar que los empleadores cuenten con herramientas de evaluación de riesgos psicosociales desarrolla el cuestionario de evaluación de riesgos psicosociales, el cual fue consensuado entre los expertos y profesionales en seguridad y salud de empresas e instituciones públicas y privadas; representantes de trabajadores y universidades, y ante el Comité Interinstitucional de Seguridad e Higiene del Trabajo, esto con la finalidad de garantizar el principio de tripartidismo y de esta forma fomentar la participación de la sociedad civil en la elaboración de políticas públicas.
El resultado global del alfa de Cronbach fue de 0,9, indica que el instrumento posee una alta fiabilidad en términos de consistencia interna ${ }^{25,26}$ y similares a los obtenidos en el método de evaluación FPSICO del Instituto Nacional de Salud e Higiene en el Trabajo $(\alpha=0,8)^{30}$, en la Guía de Identificación de Factores Psicosociales realizada en México $(\alpha=0,8)^{31}$, la traducción al castellano del Cuestionario ISTAS21 $(\alpha=0,8)^{4}$, el Cuestionario de Factores Psicosociales en el Trabajo para Perú $(\alpha=0,9)^{2}$ y el Cuestionario para Evaluar Riesgos Psicosociales en el Ambiente Laboral de Chile $(\alpha=0,9)^{10}$, así como, en otros instrumentos: Evaluación de riesgos psicosociales en el entorno laboral (cuestionario DECORE) ${ }^{16}$, Encuesta de Quebec sobre condiciones de trabajo, empleo, salud y seguridad laboral (EQCOTESST; adaptación para Chile) ${ }^{16}$ y; Primera Encuesta sobre Seguridad y Salud en el Trabajo (I-ECSST; Ecuador). ${ }^{29}$

Las dimensiones del presente cuestionario permiten valorar diferentes aspectos sobre los riesgos psicosociales incluidos en otros instrumentos de medición ${ }^{16}$. Así, pueden valorarse los requerimientos mentales y físicos del trabajador en su puesto y actividad laboral (carga y ritmo de trabajo), desarrollo de competencias; Liderazgo; Margen de Acción y Control; Organización del Trabajo; Recuperación y; Soporte y Apoyo, además otros aspectos: Acoso Discriminatorio; Acoso Laboral; Acoso Sexual; Adicción al Trabajo; Doble Presencia; Estabilidad Laboral y Emocional y aspectos relacionados con condiciones de trabajo y salud auto-percibida decir I-ECSST. ${ }^{29}$

Estos resultados se pueden evidenciar también con la Encuesta Nacional de Empleo, Desempleo y Subempleo, realizada por el Instituto Nacional de Estadísticas y Censos que en marzo 2017, indica que el 15,21\% de los trabajadores con empleo se encuentran descontentos en su trabajo debido a muchas horas de trabajo el $8,91 \%{ }^{2}$ por excesiva carga de trabajo en horarios normales, 14,88\% por las actividades o tareas que realiza, 2,56\% por el ambiente de trabajo (malas relaciones entre compañeros o jefes) y el 69.98\% por las

Table 3.

Correlaciones por dimensiones del cuestionario.

\begin{tabular}{|c|c|c|c|c|c|c|c|c|c|c|c|c|c|c|c|}
\hline & $\begin{array}{c}\text { Carga y } \\
\text { Ritmo de } \\
\text { Trabajo }\end{array}$ & $\begin{array}{l}\text { Desarrollo } \\
\text { de Compe- } \\
\text { tencias }\end{array}$ & $\begin{array}{l}0 \\
\text { - } \\
\text { Liderazgo }\end{array}$ & $\begin{array}{l}\text { Margen de } \\
\text { Acción y } \\
\text { o Control }\end{array}$ & $\begin{array}{l}\text { Organi- } \\
\text { zación del } \\
\text { Trabajo }\end{array}$ & $\begin{array}{l}\text { Recupe- } \\
\text { ración }\end{array}$ & $\begin{array}{c}\text { Soporte } \\
y \\
\text { Apoyo }\end{array}$ & $\begin{array}{c}\text { Acoso } \\
\text { Dircrimi- } \\
\text { natorio }\end{array}$ & $\begin{array}{l}\text { Acoso } \\
\text { Laboral }\end{array}$ & $\begin{array}{l}\text { Acoso } \\
\text { Sexual }\end{array}$ & $\begin{array}{c}\text { Adición } \\
\text { al } \\
\text { Trabajo }\end{array}$ & $\begin{array}{l}\text { Condi- } \\
\text { ciones de } \\
\text { Trabajo }\end{array}$ & $\begin{array}{l}\text { Doble } \\
\text { Presencia }\end{array}$ & $\begin{array}{l}\text { Estabilidad } \\
\text { a Laboral }\end{array}$ & $\begin{array}{l}\text { Salud } \\
\text { d Auto- } \\
\text { Percibida }\end{array}$ \\
\hline $\begin{array}{l}\text { Carga y } \\
\text { Ritmo de Trabajo }\end{array}$ & - & $0,502^{* *}$ & $0,530^{* *}$ & $0,512^{* *}$ & $0,516^{* *}$ & $0,582^{* *}$ & $0,478^{* *}$ & $0,483^{* *}$ & $0,519^{* *}$ & $0,310^{* *}$ & $0,524^{* *}$ & $0,404^{* *}$ & $0,410^{* *}$ & $0,552^{* *}$ & $0,480^{* *}$ \\
\hline $\begin{array}{l}\text { Desarrollo de } \\
\text { Competencias }\end{array}$ & $0,502^{* *}$ & - & $0,623^{* *}$ & $0,560^{* *}$ & $0,631^{* *}$ & $0,444^{* *}$ & $0,597^{* *}$ & $0,495^{* *}$ & $0,431^{* *}$ & $0,284^{* *}$ & $0,444^{* *}$ & $0,467^{* *}$ & $0,348^{* *}$ & $0,583^{* *}$ & $0,455^{* *}$ \\
\hline Liderazgo & $0,530^{* *}$ & $0,623^{* *}$ & - & $0,742^{* *}$ & $0,699^{* *}$ & $0,570^{* *}$ & $0,647^{* *}$ & $0,579^{* *}$ & $0,533^{* *}$ & $0,325^{* *}$ & $0,483^{* *}$ & $0,513^{* *}$ & $0,452^{* *}$ & $0,681^{* *}$ & $0,471^{* *}$ \\
\hline $\begin{array}{l}\text { Margen de } \\
\text { Acción y Control }\end{array}$ & $0,512^{* *}$ & $0,560^{* *}$ & $0,742^{* *}$ & - & $0,718^{* *}$ & $0,597^{* *}$ & $0,658^{* *}$ & $0,591^{* *}$ & $0,537^{* *}$ & $0,352^{* *}$ & $0,487^{* *}$ & $0,505^{* *}$ & $0,476^{* *}$ & $0,664^{* *}$ & $0,478^{* *}$ \\
\hline $\begin{array}{l}\text { Organización } \\
\text { del Trabajo }\end{array}$ & $0,516^{* *}$ & $0,631^{* *}$ & $0,699^{* *}$ & $0,718^{* *}$ & - & $0,593^{* *}$ & $0,745^{* *}$ & $0,636^{* *}$ & $0,543^{* *}$ & $0,385^{* *}$ & $0,537^{* *}$ & $0,593^{* *}$ & $0,470^{* *}$ & $0,705^{* *}$ & $0,509^{* *}$ \\
\hline Recuperación & $0,582^{* *}$ & $0,444^{* *}$ & $0,570^{* *}$ & $0,597^{* *}$ & $0,593^{* *}$ & - & $0,581^{* *}$ & $0,543^{* *}$ & $0,568^{* *}$ & $0,345^{* *}$ & $0,571^{* *}$ & $0,483^{* *}$ & $0,481^{* *}$ & $0,617^{* *}$ & $0,518^{* *}$ \\
\hline Soporte y Apoyo & $0,478^{* *}$ & $0,597^{* *}$ & $0,647^{* *}$ & $0,658^{* *}$ & $0,745^{* *}$ & $0,581^{* *}$ & - & $0,634^{* *}$ & $0,578^{* *}$ & $0,392^{* *}$ & $0,534^{* *}$ & $0,598^{* *}$ & $0,472^{* *}$ & $0,720^{* *}$ & $0,524^{* *}$ \\
\hline $\begin{array}{l}\text { Acoso } \\
\text { Discriminatorio }\end{array}$ & $0,483^{* *}$ & $0,495^{* *}$ & $0,579^{* *}$ & $0,591^{* *}$ & $36^{* *}$ & $0,543^{* *}$ & $0,634^{* *}$ & - & $621^{* *}$ & $0,523^{* *}$ & $0,624^{* *}$ & $0,602^{* *}$ & $0,546^{* *}$ & $0,757^{* *}$ & $0,573^{* *}$ \\
\hline Acoso Laboral & $0,519^{* *}$ & $0,431^{* *}$ & $0,533^{* *}$ & $0,537^{* *}$ & $0,543^{* *}$ & $0,568^{* *}$ & $0,578^{* *}$ & $0,621^{* *}$ & - & $0,462^{* *}$ & $0,607^{* *}$ & $0,540^{* *}$ & $0,580^{* *}$ & $0,712^{* *}$ & $0,596^{* *}$ \\
\hline Acoso Sexual & $0,310^{* *}$ & $0,284^{* *}$ & $0,325^{* *}$ & $0,352^{* *}$ & $0,385^{* *}$ & $0,345^{* *}$ & $0,392^{* *}$ & $0,523^{* *}$ & $0,462^{* *}$ & - & $0,484^{* *}$ & $0,421^{* *}$ & $0,484^{* *}$ & $0,490^{* *}$ & $0,425^{* *}$ \\
\hline Adición al Trabajo & $0,524^{* *}$ & $0,444^{* *}$ & $0,483^{* *}$ & $0,487^{* *}$ & $0,537^{* *}$ & $0,571^{* *}$ & $0,534^{* *}$ & $0,624^{* *}$ & $0,607^{* *}$ & $0,484^{* *}$ & - & $0,529^{* *}$ & $0,576^{* *}$ & $0,637^{* *}$ & $0,621^{* *}$ \\
\hline $\begin{array}{l}\text { Condiciones } \\
\text { de Trabajo }\end{array}$ & $0,404^{* *}$ & $0,467^{* *}$ & $0,513^{* *}$ & $0,505^{* *}$ & $0,593^{* *}$ & $0,483^{* *}$ & $0,598^{* *}$ & $0,602^{* *}$ & $0,540^{* *}$ & $0,421^{* *}$ & $0,529^{* *}$ & - & $0,483^{* *}$ & $0,627^{* *}$ & $0,502^{* *}$ \\
\hline Doble Presencia & $0,410^{* *}$ & $0,348^{* *}$ & $0,452^{* *}$ & $0,476^{* *}$ & $0,470^{* *}$ & $0,481^{* *}$ & $0,472^{* *}$ & $0,546^{* *}$ & $0,580^{* *}$ & $0,484^{* *}$ & $0,576^{* *}$ & $0,483^{* *}$ & - & $0,556^{* *}$ & $0,468^{* *}$ \\
\hline Estabilidad Laboral & $0,552^{* *}$ & $0,583^{* *}$ & $0,681^{* *}$ & $0,664^{* *}$ & $0,705^{* *}$ & $0,617^{* *}$ & $0,720^{* *}$ & $0,757^{* *}$ & $0,712^{* *}$ & $0,490^{* *}$ & $0,637^{* *}$ & $0,627^{* *}$ & $0,556^{* *}$ & - & $0,649^{* *}$ \\
\hline $\begin{array}{l}\text { Salud } \\
\text { Auto-Percibida }\end{array}$ & $0,480^{* *}$ & $0,455^{* *}$ & $0,471^{* *}$ & $0,478^{* *}$ & $0,509^{* *}$ & $0,518^{* *}$ & $0,524^{* *}$ & $0,573^{* *}$ & $0,596^{* *}$ & $0,425^{* *}$ & $0,621^{* *}$ & $0,502^{* *}$ & $0,468^{* *}$ & $0,649^{* *}$ & - \\
\hline
\end{tabular}

**. La correlación es significativa en el nivel 0,01 (bilateral). 


\section{Tabla 4.}

Matriz de componentes rotados.

\begin{tabular}{|c|c|c|c|c|c|c|c|}
\hline İtem & & & & Factor & & & \\
\hline $\mathrm{N}^{\circ}$ & 1 & 2 & 3 & 4 & 5 & 6 & 7 \\
\hline 1 & & & & & & & 0,426 \\
\hline 2 & & & & & & & 0,514 \\
\hline 3 & & & & & & & 0,530 \\
\hline 4 & & & & & & & 0,590 \\
\hline 5 & & & & & & 0,585 & \\
\hline 6 & & & & & & & 0,419 \\
\hline 7 & & & & 0,597 & & & \\
\hline 8 & & & & 0,522 & & & \\
\hline 9 & 0,533 & & & & & & \\
\hline 10 & 0,748 & & & & & & \\
\hline 11 & 0,678 & & & & & & \\
\hline 12 & 0,757 & & & & & & \\
\hline 13 & 0,738 & & & & & & \\
\hline 14 & 0,682 & & & & & & \\
\hline 15 & 0,500 & & & & & & \\
\hline 16 & 0,390 & & & & & & \\
\hline 17 & 0,598 & & & & & & \\
\hline 18 & 0,642 & & & & & & \\
\hline 19 & & & & 0,374 & & & \\
\hline 20 & & & & 0,558 & & & \\
\hline 21 & & & & & & 0,434 & \\
\hline 22 & & & & 0,611 & & & \\
\hline 23 & & & & 0,429 & & & \\
\hline 24 & & & & & & 0,590 & \\
\hline 25 & & & & & 0,654 & & \\
\hline 26 & & & & & 0,664 & & \\
\hline 27 & & & & & 0,650 & & \\
\hline 28 & & & & & 0,608 & & \\
\hline 29 & & & & & 0,607 & & \\
\hline 30 & & & & 0,406 & & & \\
\hline 31 & & & 0,568 & & & & \\
\hline 32 & & & & & & 0,396 & \\
\hline 33 & & & & 0,438 & & & \\
\hline 34 & & & & 0,623 & & & \\
\hline 35 & & & 0,563 & & & & \\
\hline 36 & & & & & & 0,335 & \\
\hline 37 & & & 0,590 & & & & \\
\hline 38 & & & 0,529 & & & & \\
\hline 39 & & & 0,567 & & & & \\
\hline 40 & & & & 0,425 & & & \\
\hline 41 & & 0,530 & & & & & \\
\hline 42 & & & 0,537 & & & & \\
\hline 43 & & 0,492 & & & & & \\
\hline 44 & & 0,468 & & & & & \\
\hline 45 & & 0,518 & & & & & \\
\hline 46 & & 0,570 & & & & & \\
\hline 47 & & 0,504 & & & & & \\
\hline 48 & & 0,511 & & & & & \\
\hline 49 & & 0,466 & & & & & \\
\hline 50 & & & 0,518 & & & & \\
\hline 51 & & 0,549 & & & & & \\
\hline 52 & & 0,454 & & & & & \\
\hline 53 & & 0,456 & & & & & \\
\hline 54 & & & 0,395 & & & & \\
\hline 55 & & 0,500 & & & & & \\
\hline 56 & & 0,526 & & & & & \\
\hline 57 & & 0,583 & & & & & \\
\hline 58 & & 0,442 & & & & & \\
\hline
\end{tabular}

pocas posibilidades de progresar, el 28,24\% no le han informado sobre los riesgos a los que está expuesto por desempeñar su trabajo actual, y el 40,34\% no ha recibido alguna capacitación o se ha capacitado (...) para prevenir accidentes y minimizar los riesgos a los que está expuesto en su trabajo actual.

Esta nueva propuesta para diagnosticar el nivel de riesgo psicosocial en la población trabajadora ecuatoriana no está exenta de limitaciones. Dado que en el país no existen hasta el momento instrumentos de medición, conlleva a no poder contrastar los resultados. En todo caso, la ausencia de unos valores que sirvan de referencia, para lo que todavía queda un largo camino por recorrer, no debe ser motivo para impedir el uso de este instrumento. ${ }^{32}$ Sin embargo, para futuras investigaciones se hace evidente realizar nuevos estudios confirmatorios, proporcionando en este sentido una oportunidad de mejora del cuestionario.

Por otra parte, la finalidad de la aplicación de este instrumento se basa en el diagnóstico, lo cual refuerza la necesidad del empleo de otros instrumentos de medición más específicos ${ }^{16}$, de tal manera que sea posible obtener una visión más puntual del riesgo psicosocial por dimensión y permitan diseñar programas de intervención en las empresas, cuyo papel lo debe desempeñar un especialista en psicologia en conjunto con otros actores. Igualmente, para futuros estudios surge la necesidad de elabobar una guía de apoyo para la implementación de programas de prevención de riesgos psicosociales. ${ }^{33,34}$

\section{CONCLUSIONES}

Considerando que la tarea de diseñar instrumentos validados para la medición riesgos psicosociales no es fácil en los países de Latinoamérica. ${ }^{2,35}$ En términos generales, el proceso de diseño, construcción y validación realizados, así como los resultados obtenidos, permiten recomendar el uso este cuestionario para el diagnóstico de riesgos psicosociales en empresas y organizaciones ecuatorianas de más diez trabajadores. Puede concluirse que este estudio contribuye a generar una herramienta de gran utilidad, orientando a mejorar las condiciones de trabajo para el Ecuador.

\section{Agradecimientos}

Queremos expresar nuestros agradecimientos al panel de expertos de las empresas e instituciones (públicas - privadas) y a las universidades colaboradoras por su apoyo en el desarrollo conceptual, metodológico y composición de los items del cuestionario. También agradecemos la colaboración de las empresas ecuatorianas que han participado de forma voluntaria y especialmente a todos los trabajadores y trabajadoras encuestados, sin cuya colaboración no habría sido posible desarrollar el estudio que aquí presentamos.

Financiación: El estudio fue financiado por la Dirección de Seguridad, Salud en el Trabajo y Gestión Integral de Riesgos perteneciente al Ministerio del Trabajo de Ecuador y en colaboración con la Universidad Internacional SEK.

Contribuciones de autoría: Lars T. Moreno Alestedt, y Diana I. Martínez Changuan participaron en la concepción de la idea, en el diseño del estudio y análisis estadístico e interpretación de los datos. Santiago M. Vaca Morales colaboro en el diseño muestral y trabajo de campo para la aplicación del cuestionario con apoyo de Pablo R. 
Suasnavas Bermúdez. Isabel M. Cárdenas Moncayo participo como experta en riesgos psicosociales en todas las etapas del proyecto. Antonio R. Gómez García dirigió todo el estudio y superviso todos los aspectos de su realización. Todos los autores participaron en la interpretación de los datos, revisaron los borradores del manuscrito y aprobaron su versión final.

Conflicto de intereses: Los autores declaran no tener ningún conflicto de intereses.
Aspectos éticos y legales: Todos los participantes encuestados fueron informados sobre los objetivos y finalidad del cuestionario y contestaron de manera auto-administrada en el lugar de trabajo, se contó con el debido consentimiento de los participantes. Se garantizó el anonimato y el tratamiento adecuado de los datos personales. El estudio fue aprobado y avalado por el Ministerio del Trabajo de Ecuador.
1. OIT-OMS. Factores Psicosociales en el trabajo. Naturaleza, incidencia y prevención. Informe del Comité Mixto OIT/OMS sobre medicina del trabajo; novena reunión, Ginebra; 18-24 sep 1984. Ginebra: OIT; 1986. (Serie Seguridad, Higiene y Medicina del Trabajo; 56).

2. Pando $M$, Varilla W, Aranda $C$, Elizalde $F$. Análisis factorial exploratorio del Cuestionario de factores psicosociales en el trabajo en Perú. An Fac Med (Lima). 2016; 77(4):365-371.

3. Kristensen TS, Hannerz H, Høgh A, Borg V. The Copenhagen Psychosocial Questionnaire-a tool for assessment and improvement of the psychosocial work environment. Scand J Work Environ Health. 2005; 31(6):438-49.

4. Moncada S, Llorens C, Navarro A, Kristensen TS. ISTAS21: Versión en lengua castellana del cuestionario psicosocial de Copenhague (COPSOO). Arch Prev Riesgos Labor. 2005; 8:18-29.

5. Stansfeld S, Candy B. Psychosocial work environment and mental health a meta-analytic review. Scand. J Work Environ Health. 2006; 32(6):443-62.

6. Nieuwenhuijsen K, Bruinvels D, Frings-Dresen M. Psychosocial work environment and stress-related disorders, a systematic review. Occup Med. 2010; 60(4):277-286.

7. García M, Cortés DA, Sánchez Higuera AC. Diseño, construcción y validación de un instrumento para evaluar el riesgo psicolaboral en empresas colombianas. Divers: Perspect Psicol. 2008; 4(1):37-51.

8. Alvarado $R$, Marchetti $N$, Villalón M, Hirmas M, Pastorino MS. Adaptación y análisis psicométrico de un cuestionario para evaluar riesgos psicosociales en el trabajo en Chile: versión media del COPSOQ. Rev Chil Salud Pública. 2009; 13 (1):7-16

9. Díaz A, Feldman L. Validación preliminar del cuestionario de estrés laboral en una muestra de trabajadores de la salud venezolanos. Cienc Trab. 2010; 12(36):320-323.

10. Alvarado R, Pérez-Franco J, Saavedra N, Fuentealba C, Alarcón A, Marchetti N, Aranda W. Validación de un cuestionario para evaluar riesgos psicosociales en el ambiente laboral en Chile. Rev Med Chile. 2012; 140(9):1154-1163.

11. López MR, García SA, Pando M. Factores de riesgo psicosocial y burnout en población económicamente activa de Lima, Perú. Cienc Trab. 2014; 16(51):164-169.

12. Gómez F, López A, Munuera M. Riesgos psicosociales en una Universidad de Ecuador: Mediante técnicas de Lógica Difusa. Rev Lusófona de Educação. 2016; (34):63-81.

13. Guamán MD, Bejarano BY, Gaibor IA. Los riesgos psicosociales y la satisfacción laboral: Un estudio descriptivo de corte transeccional aplicado al personal administrativo de una institución de Educación Superior en la República del Ecuador. Rev Publicando. 2017; 4(2):711-729.

14. Lara JE, Pando M. El Mobbing y los Sintomas de Estrés en Docentes Universitarios del Sector Público. Cienc Trab. 2014; 16(49):43-48.

15. Kompier M. Assessing the psychosocial work environment "subjective" versus "objective" measurement. Scand. J Work Environ Health. 2005; 31(6):405-408.

16. Charria V, Sarsosa K, Arenas F. Factores de riesgo psicosocial laboral: métodos e instrumentos de evaluación. Rev Fac Nac Salud Pública 2011; 29(4):380-391.

17. Ecuador. Ministerio del Trabajo. Decreto Ejecutivo 2393 Reglamento de Seguridad y Salud de los Trabajadores y Mejoramiento del Medio Ambiente de Trabajo. Quito: Ministerio del Trabajo; 1986.
18. Organización Internacional de Trabajo-OIT. Convenio 111. Convenio sobre la discriminación (empleo y ocupación). 42a Reunión CIT; 25 jun 1958; Ginebra, Suiza. Ginebra: OIT; 1960.

19. Ecuador. Ministerio del Trabajo. Ley Orgánica de Justicia Laboral y Reconocimiento del Trabajo en el Hogar. Quito: Ministerio del Trabajo; 2015.

20. Ecuador. Ministerio de Trabajo y Empleo. Acuerdo Ministerial 0398 Prohibición de terminación de la relación laboral a personas con VIH-SIDA. Quito: Ministerio del Trabajo; 2006.

21. Ecuador. Ministerio del Trabajo. Acuerdo Ministerial 082 Normativa de erradicación de la discriminación en el ámbito laboral. Quito: Ministerio del Trabajo; 2017.

22. Instituto Nacional de Estadística y Censos. Directorio de Empresas [en línea]. Quito: INEC; 2016 [citado jul 2018]. Disponible en: http://www.ecuadorencifras.gob.ec/directoriodeempresas

23. Instituto Nacional de Estadística y Censos. Clasificación Nacional de Actividades Económicas [en línea]. Quito: INEC; 2012 [citado jul 2018]. Disponible en: http://aplicaciones2.ecuadorencifras.gob.ec/SIN/descargas/ciiu. pdf

24. Comunidad Andina de Naciones. Decisión 702: Sistema Andino de Estadistica de la PYME. Lima: CAN; 2008.

25. Feldt LS, Woodruff DJ, Salih FA. Statistical inference for coefficient alpha. Appl Psych Meas. 1987; 11(1):93-103.

26. Bonett DG. Sample size requirements for testing and estimating coefficient alpha. J Educat Behav Statist. 2002; 27:335-340.

27. Carvajal A, Centeno C, Watson R, Martínez M, Sanz RA. ¿Cómo validar un instrumento de medida de la salud? An Sist Sanit Navar. 2011; 34:63-72.

28. Romani $F$, Márquez J, Wong P. Uso de los métodos estadisticos en artículos originales de cinco revistas biomédicas peruanas; periodo 2002-2009. Rev Peru Epidemiol. 2010; 14:1-8.

29. Gómez AR. I Encuesta sobre Seguridad y Salud en el Trabajo para Ecuador: I-ESST. CienciAmérica. 2017; 6(1):67-75.

30. Ferrer Puig R, Guilera Ferré G, Peró Cebollero M. Propiedades psicométricas del instrumento de valoración de riesgos psicosociales del Instituto Nacional de Seguridad e Higiene en el Trabajo (FPSICO). Barcelona: Universidad de Barcelona; 2011

31. Aranda $C$, Pando $M$, Ronquillo J. Confiabilidad y validez de una guía de identificación sobre factores psicosociales en trabajadores mexicanos de diversos giros laborales. Rev Iber Psic CyT (Bogotá). 2014; 7(1):25-38.

32. Benavides FG, Gimeno D, Benach J, Martínez JM, Jarque S, Berra A. et al. Descripción de los factores de riesgo psicosocial en cuatro empresas. Gac Sanit. 2002; 16(3):222-229.

33. Navarro AJ. Modelo de intervención psicosocial en las organizaciones frente al estrés laboral: estrategia operativa. Med Segur Trab. 2018; 55(215):86-98.

34. Polo JD, Palacio JE, De Castro AM, Mebarak MR, Velilla JL. Riesgos psicosociales: la psicología organizacional positiva como propuesta de promoción, prevención e intervención. Salud (Barranquilla). 2013; 29(3):561-575.

35. Benavides FG, Benach J, Muntaner C. Psychosocial risk factors at workplace: is there enough evidence to establish reference values? J Epidemiol Commun $\mathrm{H}$. 2002; 56:244-245. 
Anexo 1. Cuestionario.

\section{INSTRUCCIONES PARA COMPLETAR EL CUESTIONARIO:}

1. Se recomienda leer detenidamente cada pregunta y opciones de respuesta antes de responder.

2. Utilice esfero para marcar la respuesta.

3. El cuestionario está diseñado para identificar y valorar todas aquellas condiciones de trabajo del ámbito psicosocial que pueden representar un riesgo para la salud y el bienestar laboral.

4. El cuestionario valora la exposición a diferentes dimensiones psicosociales derivados de la organización del trabajo.

5. Todas las preguntas deben ser contestadas, éstas tienen varias opciones de

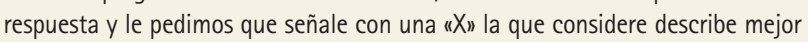
su situación.

6. Responda sinceramente, recuerde que el cuestionario es anónimo y se garantiza la confidencialidad de las respuestas.
7. Tenga presente que no existen respuestas correctas o incorrectas.

8. La aplicación total del cuestionario demora alrededor de 15 a 20 minutos.

9. Se sugiere no conversar mientras responde el cuestionario para que se pueda concentrar, recuerde que su opinión es importante.

10. Utilice el espacio de observaciones y sugerencia para cualquier comentario respecto a este cuestionario.

11. Cada uno de los items de respuesta cuenta con un número, el cual debe ser sumado en cada dimensión, la sumatoria de éstos permitirá interpretar las dimensiones psicosociales de su cuestionario. En la última página encontrará las instrucciones para la interpretación.

12. Si usted no entiende alguna pregunta o necesita apoyo, solicite al facilitador.

Muchas gracias por su colaboración

\begin{tabular}{|c|c|c|c|c|}
\hline & \multicolumn{4}{|c|}{ Respuestas } \\
\hline & $\begin{array}{l}\text { Completamente } \\
\text { de Acuerdo }\end{array}$ & $\begin{array}{l}\text { Respues } \\
\text { Parcialmente } \\
\text { de Acuerdo }\end{array}$ & \multirow[t]{2}{*}{$\begin{array}{l}\text { Poco de } \\
\text { Acuerdo }\end{array}$} & $\begin{array}{c}\text { En } \\
\text { Desacuerdo }\end{array}$ \\
\hline \multicolumn{4}{|l|}{ CARGA Y RITMO DE TRABAJO } & \\
\hline $\begin{array}{l}\text { 1. Considero que son aceptables las solicitudes y requerimientos que me piden otras personas } \\
\text { (compañeros de trabajo, usuarios, clientes). }\end{array}$ & {$[4]$} & [3] & {$[2]$} & {$[1]$} \\
\hline 2. Decido el ritmo de trabajo en mis actividades. & {$[4]$} & [3] & {$[2]$} & {$[1]$} \\
\hline 3. Las actividades y/o responsabilidades que me fueron asignadas no me causan estrés. & [4] & [3] & {$[2]$} & {$[1]$} \\
\hline $\begin{array}{l}\text { 4. Tengo suficiente tiempo para realizar todas las actividades que me han sido encomendadas } \\
\text { dentro de mi jornada laboral. }\end{array}$ & [4] & [3] & {$[2]$} & {$[1]$} \\
\hline \multicolumn{5}{|c|}{ Sumatoria de puntos de la Dimensión [................] } \\
\hline \multicolumn{5}{|l|}{ DESARROLLO DE COMPETENCIAS } \\
\hline $\begin{array}{l}\text { 5. Considero que tengo los suficientes conocimientos, habilidades y destrezas para desarrollar el trabajo } \\
\text { para el cual fui contratado. }\end{array}$ & [4] & [3] & [2] & [1] \\
\hline 6. En mi trabajo aprendo y adquiero nuevos conocimientos, habilidades y destrezas de mis compañeros de trabajo. & [4] & [3] & [2] & {$[1]$} \\
\hline $\begin{array}{l}\text { 7. En mi trabajo se cuenta con un plan de carrera, capacitación y/o entrenamiento para el desarrollo } \\
\text { de mis conocimientos, habilidades y destrezas. }\end{array}$ & [4] & [3] & [2] & {$[1]$} \\
\hline $\begin{array}{l}\text { 8. Tengo suficiente tiempo para realizar todas las actividades que me han sido encomendadas } \\
\text { dentro de mi jornada laboral. }\end{array}$ & [4] & [3] & {$[2]$} & {$[1]$} \\
\hline \multicolumn{5}{|c|}{ Sumatoria de puntos de la Dimensión [................] } \\
\hline \multicolumn{5}{|l|}{ LIDERAZGO } \\
\hline 9. En mi trabajo se reconoce y se da crédito a la persona que realiza un buen trabajo o logran sus objetivos. & [4] & [3] & [2] & [1] \\
\hline 10. Mi jefe inmediato esta dispuesto a escuchar propuestas de cambio e iniciativas de trabajo. & [4] & [3] & [2] & {$[1]$} \\
\hline 11. Mi jefe inmediato establece metas, plazos claros y factibles para el cumplimiento de mis funciones o actividades. & {$[4]$} & [3] & {$[2]$} & {$[1]$} \\
\hline 12. Mi jefe inmediato interviene, brinda apoyo, soporte y se preocupa cuando tengo demasiado trabajo que realizar. & [4] & [3] & {$[2]$} & {$[1]$} \\
\hline 13. Mi jefe inmediato me brinda suficientes lineamientos y retroalimentación para el desempeño de mi trabajo. & [4] & [3] & [2] & [1] \\
\hline 14. Mi jefe inmediato pone en consideración del equipo de trabajo, las decisiones que pueden afectar a todos. & [4] & [3] & [2] & [1] \\
\hline
\end{tabular}

MARGEN DE ACCIÓN Y CONTROL

15. En mi trabajo existen espacios de discusión para debatir abiertamente los problemas comunes y diferencias de opinión.

[4] [3] [2] [1]

16. Me es permitido realizar el trabajo con colaboración de mis compañeros de trabajo y/u otras áreas.

17. Mi opinión es tomada en cuenta con respecto a fechas límites en el cumplimiento de mis actividades o cuando exista cambio en mis funciones.

[4]

[1]

[4]

[4]

[2] [1]

[1] [1]

\section{ORGANIZACIÓN DEL TRABAJO}

19. Considero que las formas de comunicación en mi trabajo son adecuados, accesibles y de fácil comprensión.

20. En mi trabajo se informa regularmente de la gestión y logros de la empresa o institución a todos los trabajadores y servidores.

21. En mi trabajo se respeta y se toma en consideración las limitaciones de las personas con discapacidad para la asignación de roles y tareas.

22. En mi trabajo tenemos reuniones suficientes y significantes para el cumplimiento de los objetivos.

23. Las metas y objetivos en mi trabajo son claros y alcanzables.

24. Siempre dispongo de tareas y actividades a realizar en mi jornada y lugar de trabajo.

\begin{tabular}{llll}
{$[4]$} & {$[3]$} & {$[2]$} & {$[1]$} \\
{$[4]$} & {$[3]$} & {$[2]$} & {$[1]$} \\
{$[4]$} & {$[3]$} & {$[2]$} & {$[1]$} \\
\hline 4$]$ & {$[3]$} & {$[2]$} & {$[1]$} \\
\hline 4$]$ & {$[3]$} & {$[2]$} & {$[1]$} \\
\hline 4$]$ & {$[3]$} & {$[2]$} & {$[1]$} \\
Sumatoria de puntos de la Dimensión $[\ldots \ldots \ldots \ldots \ldots .]$.
\end{tabular}




\section{RECUPERACIÓN}

\begin{tabular}{cccc}
\multicolumn{4}{c}{ Respuestas } \\
Completamente & $\begin{array}{c}\text { Parcialmente } \\
\text { Poco de }\end{array}$ & En \\
de Acuerdo & de Acuerdo & Acuerdo & Desacuerdo
\end{tabular}

25. Después del trabajo tengo la suficiente energía como para realizar otras actividades. de Acuerdo de Acuerdo Acuerdo esacuerdo

26. En mi trabajo se me permite realizar pausas de periodo corto para renovar y recuperar la energía.

27. En mi trabajo tengo tiempo para dedicarme a reflexionar sobre mi desempeño en el trabajo.

28. Tengo un horario y jornada de trabajo que se ajusta a mis expectativas y exigencias laborales.

29. Todos los días siento que he descansado lo suficiente y que tengo la energía para iniciar mi trabajo.

$\begin{array}{llll}{[4]} & {[3]} & {[2]} & {[1]} \\ {[4]} & {[3]} & {[2]} & {[1]} \\ {[4]} & {[3]} & {[2]} & {[1]} \\ {[4]} & {[3]} & {[2]} & {[1]} \\ {[4]} & {[3]} & {[2]} & {[1]}\end{array}$

\section{SOPORTE Y APOYO}

30. El trabajo está organizado de tal manera que fomenta la colaboración de equipo y el diálogo con otras personas. [4]

31. En mi trabajo percibo un sentimiento de compañerismo y bienestar con mis colegas.

32. En mi trabajo se brinda el apoyo necesario a los trabajadores sustitutos o trabajadores con algún grado de discapacidad y enfermedad.

33. En mi trabajo se me brinda ayuda técnica y administrativa cuando lo requiero.

34. En mi trabajo tengo acceso a la atención de un médico, psicólogo, trabajadora social, consejero, etc. en situaciones de crisis y/o rehabilitación.

$\begin{array}{llll}{[4]} & {[3]} & {[2]} & {[1]} \\ {[4]} & {[3]} & {[2]} & {[1]} \\ {[4]} & {[3]} & {[2]} & {[1]} \\ {[4]} & {[3]} & {[2]} & {[1]} \\ {[4]} & {[3]} & {[2]} & {[1]}\end{array}$

\section{OTRAS DIMENSIONES}

Sumatoria de puntos de la Dimensión [...............]

35. En mi trabajo tratan por igual a todos, indistintamente la edad que tengan.

36. Las directrices y metas que me autoimpongo, las cumplo dentro de mi jornada y horario de trabajo.

38. Tengo un trabajo donde los hombres y mujeres tienen las mismas oportunidades.

39. En mi trabajo me siento aceptado y valorado.

40. Los espacios y ambientes fisicos en mi trabajo brindan las facilidades para el acceso de las personas con discapacidad.

41. Considero que mi trabajo esta libre de amenazas, humillaciones, ridiculizaciones, burlas, calumnias o difamaciones reiteradas con el fin de causarme daño.

42. Me siento estable a pesar de cambios que se presentan en mi trabajo.

43. En mi trabajo estoy libre de conductas sexuales que afecten mi integridad física, psicológica y moral.

44. Considero que el trabajo que realizo no me causa efectos negativos a mi salud fisica y mental.

45. Me resulta fácil relajarme cuando no estoy trabajando.

46. Siento que mis problemas familiares o personales no influyen en el desempeño de las actividades en el trabajo.

47. Las instalaciones, ambientes, equipos, maquinaria y herramientas que utilizo para realizar el trabajo son las adecuadas para no sufrir accidentes de trabajo y enfermedades profesionales.

48. Mi trabajo esta libre de acoso sexual.

49. En mi trabajo se me permite solucionar mis problemas familiares y personales.

50. Tengo un trabajo libre de conflictos estresantes, rumores maliciosos o calumniosos sobre mi persona.

51. Tengo un equilibrio y separo bien el trabajo de mi vida personal.

52. Estoy orgulloso de trabajar en mi empresa o institución.

53. En mi trabajo se respeta mi ideología, opinión política, religiosa, nacionalidad y orientación sexual.

54. Mi trabajo y los aportes que realizo son valorados y me generan motivación.

55. Me siento libre de culpa cuando no estoy trabajando en algo.

56. En mi trabajo no existen espacios de uso exclusivo de un grupo determinado de personas ligados a un privilegio, por ejemplo, cafetería exclusiva, baños exclusivos, etc., mismo que causa malestar y perjudica mi ambiente laboral.

57. Puedo dejar de pensar en el trabajo durante mi tiempo libre (pasatiempos, actividades de recreación, otros).

58. Considero que me encuentro física y mentalmente saludable.
[4]

[4]

[4]

[4]

[3] [2] [1]

[3]

[3]

[3]

[3]

[3]

[2]

[2]

[2] [1]

[4]

[4]

[4] [3] [2] [1]

[2]

[2]

[1]

[4] [3] [2] [1]

[4] [3] [2] [1]

[4] [3]

[4] [3]

[4] [3]

[2]

[1]

[2]

[2]

[4] [3] [2] [1]

$[4] \quad[3] \quad[2] \quad[1]$

[4] [3] [2] [1]

$[4] \quad[3] \quad[2] \quad[1]$

[4] [3] [2] [1]

[4] [3] [2] [1]

$[4] \quad[3] \quad[2] \quad[1]$

$[4]$

[4] [3]

[3]

[2]

[1]

[4] [3] [2] [1]

[4] [3] [2] [1]

[4] [3] [2] [1]

Sumatoria de puntos de la Dimensión [............... 
Anexo 2. Puntuaciones y Nivel de Riesgo Psicosocial según Dimensiones.

\begin{tabular}{|c|c|c|c|c|}
\hline DIMENSIONES & & $\begin{array}{c}\text { RIESGO } \\
\text { BAJO }\end{array}$ & MEDIO & ALTO \\
\hline CARGA Y RITMO DE TRABAJO & & {$[13$ a 16$]$} & [8 a 12] & {$[4$ a 7$]$} \\
\hline DESARROLLO DE COMPETENCIAS & & {$[13$ a 16$]$} & [8 a 12] & {$[4$ a 7$]$} \\
\hline LIDERAZGO & & [18 a 24] & [12 a 17] & [6 a 11] \\
\hline MARGEN DE ACCIÓN Y CONTROL & & [13 a 16] & [8 a 12] & {$[4$ a 7$]$} \\
\hline ORGANIZACIÓN DEL TRABAJO & & [18 a 24] & [12 a 17] & {$[6$ a 11$]$} \\
\hline RECUPERACIÓN & & [16 a 20] & [10 a 15] & [5 a 9] \\
\hline SOPORTE Y APOYO & & [16 a 20] & [10 a 15] & {$[5$ a 9$]$} \\
\hline \multirow[t]{3}{*}{ OTROS DIMENSIONES * } & & [73 a 96] & [49 a 72$]$ & [24 a 48] \\
\hline & Nivel de Riesgo Global & [175 a 232] & [117 a 174] & [58 a 116] \\
\hline & Color & Verde & Amarillo & Rojo \\
\hline
\end{tabular}

* Acoso Discriminatorio; Acoso Laboral; Acoso Sexual; Adicción al Trabajo; Condiciones de Trabajo; Doble Presencia; Estabilidad Laboral y Emocional y; Salud Auto-percibida.

Anexo 3. Interpretación de los Niveles de Riesgo Psicosocial por Dimensión y Global.

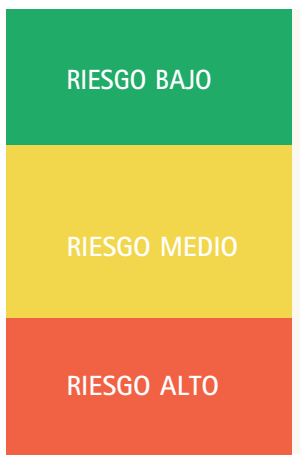

El riesgo es de impacto potencial mínimo sobre la seguridad y salud, no genera a corto plazo efectos nocivos. Estos efectos pueden ser evitados a través de un monitoreo periódico de la frecuencia y probabilidad de que ocurra y se presente una enfermedad ocupacional, las acciones irán enfocadas a garantizar que el nivel se mantenga.

El riesgo es de impacto potencial moderado sobre la seguridad y salud puede comprometer las mismas en el mediano plazo, causando efectos nocivos para la salud, afectaciones a la integridad física y enfermedades ocupacionales. En caso de que no se aplicaren las medidas de seguridad y prevención correspondientes de manera continua y conforme a la necesidad específica identificada, los impactos pueden generarse con mayor probabilidad y frecuencia.

El riesgo es de impacto potencial alto sobre la seguridad y la salud de las personas, los niveles de peligro son intolerables y pueden generar efectos nocivos para la salud e integridad física de las personas de manera inmediata. Se deben aplicar las medidas de seguridad y prevención de manera continua y conforme a la necesidad especifica identificada para evitar el incremento a la probabilidad y frecuencia. 